University of Nebraska - Lincoln

DigitalCommons@University of Nebraska - Lincoln

Robert Katz Publications

Research Papers in Physics and Astronomy

September 1991

\title{
Track physics model of radiation effects
}

Robert Katz

University of Nebraska-Lincoln, rkatz2@unl.edu

Follow this and additional works at: https://digitalcommons.unl.edu/physicskatz

Part of the Physics Commons

Katz, Robert, "Track physics model of radiation effects" (1991). Robert Katz Publications. 125.

https://digitalcommons.unl.edu/physicskatz/125

This Article is brought to you for free and open access by the Research Papers in Physics and Astronomy at DigitalCommons@University of Nebraska - Lincoln. It has been accepted for inclusion in Robert Katz Publications by an authorized administrator of DigitalCommons@University of Nebraska - Lincoln. 


\title{
Track physics model of radiation effects
}

\author{
Robert Katz, University of Nebraska-Lincoln
}

Published in:

RADECS 91--Radiation Effects on Computation and Systems: First European Conference on Radiation and its Effects on Devices and Systems : La Grande-Motte, near Montpellier, France, September 9-12,1991 (European Space Agency, Institute of Electrical and Electronics Engineers, \& Université des sciences et techniques du Languedoc, 1992). [ISBN: 0-7803-0208-7] Pages: 558 - 560

Online at http://ieeexplore.ieee.org/xpl/tocresult.jsp?isnumber=5570\&isYear=1991 Article @ http://ieeexplore.ieee.org/xpls/abs all.jsp?isnumber=5570\&arnumber=213538\&count=110\&index=7

Digital Object Identifier: 10.1109/RADECS.1991.213538

\begin{abstract}
Radiation effects induced by heavy ions in many materials with diverse end points are well described by the conceptual structure and equations of track physics, first developed for heavy ion tracks in nuclear emulsions. The model describes scintillators, biological cell inactivation and mutation, radiation chemistry, latent tracks in insulators, the response of resists to heavy ions, and other systems. A detector is taken to be composed of small targets whose response to ionizing radiation is principally to secondary electrons. The response is calibrated through determination of the probability of target (in)activation as a function of the absorbed dose of $\gamma$ rays. This is then translated into the radial distribution of the probability of target (in)activation about the path of heavy ion through knowledge of the radial distribution of dose from $\delta$ rays. Radial integration yields the action cross-section $\sigma$, from which the response as a function of fluence of heavy ions is calculated. The author asks whether the track physics model is needed to describe single event upsets, or the effects produced in bulk matter by HZE particles.
\end{abstract}




\title{
Track Physics Model of Radiation Effects
}

\author{
Robert Katz \\ University of Nebraska, \\ Lincoln, NE 68588-0111 USA \\ Telephone (1) 4024722779 \\ Fax (1) 4024722879
}

\begin{abstract}
Radiation effects induced by heavy ions in many materials with diverse end points are well described by the conceptual structure and equations of track physics, first developed for heavy ion tracks in nuclear emulsions. The model describes scintillators, biological cell inactivation and mutation, radiation chemistry, latent tracks in insulators, the response of resists to heavy ions, and other systems. A detector is taken to be composed of small targets whose response to ionizing radiation is principally to secondary electrons. The response is calibrated through determination of the probability of target (in)activation as a function of the absorbed dose of $\gamma$ rays. This is then translated into the radial distribution of the probability of target (in)activation about the path of a heavy ion through knowledge of the radial distribution of dose from $\delta$ rays. Radial integration yields the action cross-section $\sigma$, from which the response as a function of fluence of heavy ions is calculated. Typically when responses differ to bombardments with different ions at the same absorbed dose and at the same LET, track structure plays a determining role. We ask whether the track physics model is needed to describe single event upsets, or the effects produced in bulk matter by HZE particles.
\end{abstract}

\section{INTRODUCTION}

Particle tracks in emulsion $[1,2,3,4]$ serve as a prototype for radiation effects from $\mathrm{HZE}$ ions in many different detecting systems. These tracks, principally caused by $\delta$ rays ejected from the medium by the passing ion, can look like a hairy rope more than $100 \mu \mathrm{m}$ wide in a sensitive emulsion, or like a string of single developed grains in another, from the same ion. Here the key question is "hittedness", nominally the number of electrons which must pass through an emulsion grain to create the "latent image". When the hittedness exceeds 1 , we do not see the tracks of individual $\delta$ rays, even though these are the cause of the effect. The appearance of a particle track can be related to the "action cross section", for this can be defined as the radial integral of the probability for activating an emulsion grain. When the track resembles a hairy rope the cross section can be many orders of magnitude greater than the physical cross sectional area of an undeveloped grain.

\footnotetext{
${ }^{1}$ This research is sponsored by the U. S. Department of Energy.
}

When the track resembles a string of beads the cross section can be significantly less than the area of a grain, for the number of activated grains per unit path length is $n=\sigma N$, where $\mathbf{N}$ is the number of undeveloped grains per unit volume. In these two cases the observed effect, the number of developable grains per unit path length, does not scale simply in the two emulsions. Similar problems are noted in radiobiology and in other detectors. Thus while the response of a scintillator is proportional to the energy of an absorbed electron, this is not the case for an absorbed ion.

\section{THE TRACK PHYSICS MODEL}

To describe the response of a detector to HZE particles we require information about the radial dose distribution abu the ion's path, from $\delta$ rays, and about the response of the detector to $\gamma$ rays as a function of dose (absorbed energ: density). This is frequently cast in the form of the cumulative Poisson distribution $\mathrm{P}(\mathrm{C}, \mathrm{A})$, where $\mathrm{C}$ is the minimal number of hits required to activate the detector, and $A$ is the mean number of hits per target. For radiation detectors we define $E_{0}$ as the dose of $\gamma$ rays at which there is an average of 1 hit per target, so that at dose $D, A=D / E_{0}$ Most common are 1-or-more hit detectors in which it is possible for a single electron passing through a target to activate it. Such detectors display exponential response to al: radiations, regardless of the mechanism of their interaction with radiation or the variety of intervening steps between the absorption of radiation and the expression of the end point. At first glance it seems improbable that detectors with entirely different response mechanisms should be described by the same mathematical form, but with different parameters [5]. But the only requirement is that the response should be initiated by the transit of a single electron of appropriate energy through the target volume. In a parallel context we may describe the exponential absorption of photons in matter as 1-hit in space, while exponential radioactive decay can be described as 1-hit in time.

The dose $\mathrm{E}_{0}$ is an average concept, for with $\gamma$ radiation the medium is exposed to a nearly random tangle of electron paths, with possibly several paths passing through a single target at high dose. It can be a large number for small 1-hit targets like virus molecules for the medium must contam many such electron tracks even to pass through $63 \%$ of these But there may be a further requirement on the energy "lose" 
by the electron in these transits for initiation of the effect. Both size and energy considerations contribute to the numerical value of $E_{0}$. Note that we do not here speak of the energy "deposited in a molecule" for this has an unsatisfactory vagueness, though the term is often used to represent the product of the dose (energy per unit mass) by the molecular mass.

Our identification of 1- or of many-hit systems is based on the comparison of the form of the cumulative Poisson distribution with the dose-response curve observed after $\gamma$ irradiation. In most systems response to heavy ions is coupled to response to $\gamma$ rays. Possible exceptions may arise when several electrons are required to pass through a target in a time short compared to the duration of a $\gamma$ ray exposure [6]. Nuclear emulsions may be so desensitized as not to reveal the tracks of fission fragments. Yet these emulsions will respond to intense $x$-ray exposures [7].

To make the transition from the response to $\gamma$ rays to the response to HZE ions we make use of knowledge of the radial distribution of dose about an ion's path $[8,9]$, as determined by Monte Carlo calculations and by experiment. We interpret the exposure to the average radial distribution of dose in a cylindrical shell whose axis is the ion's path as if there were a macroscopic volume made up of the material in many such shells about many ions. The probability of (in)activation of a target in such a shell is then determined from the experimental finding from a $\gamma$ ray exposure at the same dose. In this way we obtain the probability $P(t)$ for target inactivation at radial distance $t$ about the path of an averaged ion. The radial integral of this probability is the action cross section $\sigma(z, \beta)$, a function of the effective charge $z$ of the ion and its speed. At low fluence where the $\delta$ rays from different ions do not overlap, $\sigma$ determines the response of the system, exponentially, for the fraction of targets activated (or killed) is $1-\exp (-\sigma F)$. In track theory we call this response "ion-kill", an "intra-track" effect.

At high fluence, and for many hit detectors, it is possible that additional damage is done by overlapping $\delta$ rays from several ions, especially in the "grain count regime" where the track has the appearance of "beads on a string". Then the targets in the gaps between beads may be partially damaged by one passing ion, though insufficiently to express the end point. In this case $\delta$ rays from other ions may contribute to the observed response. We term this response " $\gamma$-kill", an "inter-track" effect. We apportion the total dose into two fractions, one part of which is assigned to ion-kill, and the other to $\gamma$-kill. The former yields exponential response with fluence. The latter yields the same response form as observed with $\gamma$ rays. We imagine that survivors of the ionkill mode represent the initial population of the $\gamma$-kill mode. The ultimate surviving fraction is then taken to be the product of the ion-kill and the $\gamma$-kill survival probabilities.

Our first task in the application of this model is to measure the parameters of any detector. These are evaluated from the measured response to $\gamma$ rays and to a series of bombardments with HZE ions, of the widest possible dynamic range. The experimental response is then fitted to formulas of the model. Typically for simply structured detectors we require knowledge of the hittedness $C$, the characteristic dose of $\gamma$ rays $E_{0}$, and the target size (though the latter is often masked and not needed in systems where all targets are damaged within 3 target radii of the ion's path).

Biological cells are more complex for there are critical targets, like chromosomes or DNA complexes contained within the cell nucleus. Here we require two size parameters, one descriptive of the target and the other descriptive of the cell nucleus. In place of the cumulative Poisson distribution we represent the response of cells through a model which assumes that a group of $m$ targets must be inactivated, each of which requiring a single hit. Thus while simply constructed systems (like nuclear emulsions) require 3 parameters, biological cells require 4 , namely $E_{0}, m$ (the number of targets), $\sigma_{0}$ ( the size of the nucleus) and $\kappa$ (a combination of $\mathrm{E}_{0}$ and $\mathrm{a}_{0}$, the internal target radius).

Once these parameters are measured, the model makes it possible to calculate response to any arbitrary radiation field whose photon dose and particle-energy spectrum are known.

Equations of the model are given elsewhere [10]. Here we describe its logical structure qualitatively. We must emphasize that the model is applicable only to detectors whose response arises from electronic interactions. Nuclear collisions contribute to that response only through their fragments. A clear indication that this model must be considered is the experimental finding that a particular end point is multiply valued in LET, arising from bombardments of different charge and speed, for then track structure clearly plays a role in determining response.

\section{RESULTS}

Through this model we have been able to describe the behavior of many physical, chemical, and biological systems.

\section{A. Nuclear Emulsions}

The track model describes: the blackening of emulsion with electron beams [11], grain count and photometric measurement of track width [12], and the computer simulation of particle tracks in emulsions of different sensitivities [3].

\section{B. Physical Detectors}

The variation in response of inorganic scintillators [13], of TLD's [14], of alanine [15] are all described as 1-hit detectors. There are other apparent 1-hit effects in solids, like color center formation, and the generation of latent tracks in CR-39 [16]. Some photoresists respond to heavy ion bombardment as many-target systems, following our formulation of radiobiological effects [17]. 


\section{Chemical Detectors}

The Fricke Dosimeter [18] behaves as a 1-hit system, while the generation of $\mathrm{HO}_{2}$ radicals in water and of $\mathrm{H}_{2}$ in benzene [19] behave like 2-hit systems.

\section{Cell Killing, Chromosome Aberration, Mutations}

Many (but not all) cell lines respond well to our radiobiological models of track structure in regard to cellular inactivation [10] in track segment irradiation with heavy ions. We have predicted the effect of "thin-down" the decline in $\sigma$ with an increase in LET for slowing HZE ions in biological experiments [20]. Cellular radiosensitivity parameters extracted from such measurements are used in conjunction with knowledge of secondary fragments from neutron and HZE beams to calculate the response of cells to such irradiations, in good agreement with experiments [21]. Parameters have been extracted from data on cell transformation and mutation induction are being used to predict response in complex fields [22]. We have recently shown that E. Coli B survives heavy ion irradiation as a 1-hit detector [23], by analysis of existing data. We have similarly shown that both single and double strand breaks in SV40 DNA in EO buffer (which emphasizes the indirect effect) also follow the pattern calculated for 1-hit detectors [24]. We have calculated the response of cells to proton beams and have shown that when the measured RBE exceeds one, this arises from target fragmentation.

\section{DISCUSSION}

Many of the effects observed after the irradiation of matter with beams of HZE particles can be accommodated within the framework of track theory, in which the description of the radiation is mechanistic, but the response of the detector to radiation is parametric and phenomenological. For no condensed matter detector is there a clear quantitative, mechanistic ab-initio explanation of its response to radiation. From the viewpoint of track physics the place for such an explanation is the response to $\gamma$ rays, for once this is known the response to HZE particles can be described by track theory. When experimentally we find that the response varies, to different HZE particles at the same LET, track structure is implicated. We can expect this to be the case when the target grains are small, of order $1 \mu \mathrm{m}$ or less, the detector is sensitive, and the charge of the incident ion is high. This can be spelled out quantitatively through track theory. The response of detectors is dominated by statistical considerations.

Track theory has global implications.

It is for this reason that we ask whether this model appears to be applicable to single event upsets with HZE particles, and to other experiments in which beams of heavy ions alter the properties of condensed matter.

\section{REFERENCES}

[1] C. F. Powell, P. H. Fowler, and D. H. Perkins, The Study of Elementary Particles by the Photographic Method. Pergamon Press, New York. 1959.

[2] R. Katz and E. J. Kobetich, "Particle Tracks in Emulsion,"Phys. Rev. vol. 186, pp. 344-351, 1969.

[3] R. Katz and F. E. Pinkerton, "Response of Nuclear Emulsion to Ionizing Radiations," Nucl. Instr. Meth. vol. 130, pp. 105-119, 1975.

[4] R. Katz, A. S-F. Li, Y-L. Chang, R. L. Rosman, and E. V. Benton, "Tracks of Argon lons in Ilford K-Series Nuclear Track Detectors," in Tenth International Conference on Solid State Nuclear Track Detectors, Pergamon Press, N.Y. 1980.

[5] R. Katz, "Track Structure Theory in Radiobiology and Radiation Detection," vol. 2, pp. 1-28, 1978.

[6] N. Itoh, "Defect Formation in Insulators under Dense Electronic Excitation," Radiat. Effects and Defects in Solids, vol. 110, pp. 19-25, 1989.

[7] L. Larsson, F. E. Pinkerton, and R. Katz, "Supralinearity in Nuclear Research Emulsions," Radiat. Effects, vol. 34, pp. 15-24, 1977.

[8] M. P. R. Waligorski, R. N. Hamm, and R. Katz, "The Radial Distribution of Dose around the path of a Heavy Ion in Liquid Water," Nucl. Tracks Radiat. Meas. vol. 11, pp. 309-319, 1986.

[9] R. Katz, K. S. Loh, Daling Luo, and G. R. Huang, "An Analytical Representation of the Radial Distribution of Dose from Heavy Ions in Water, LiF, NaI, and $\mathrm{SiO}_{2}$ Radiat. Effects and Defects in Solids, vol. 114. pp. 15-20, 1990.

[10] R. Katz, S. C. Sharma, and M. Homayoonfar, "The Structure of Particle Tracks," in Topics in Radiation Dosimetry, Suppl. 1, F. H. Attix, ed., Academic Press, New York, 1972.

[11] R. Katz and E. J. Kobetich, "Response of Nuclear Emulsion to Electron Beams," Nucl. Instr. Meth. vol. 79, pp. 320-324, 1970.

[12] M. Jensen and $O$. Mathiesen, "Measured and Calculated Absorptance of Tracks of Fast Heavy Ions in Ilford G5 Nuclear Emulsion," Physica Scripta, vol. 13, pp. 75-82, 1976.

[13] R. Katz and E. J. Kobetich, "Response of NaI(TI) to Energetic Heavy Ions," Phys. Rev. vol. 170, pp. 397-400, 1968.

[14] Daling Luo and R. Katz, "Application of Track Structure Theory to the Response of TLD-100 to Energetic Heavy Ions, "to be published.

[15] M. P. R. Waligorski, G. Danialy, K. S. Loh, and R. Katz, "Response of the Alanine Dosimeter to Charged Particle and Neutron Iradiations," Appl. Radial. and lsotopes vol. 40, pp. 923-933, 1989.

[16] R. Katz, "Formation of Etchable Tracks in Plastics." Nucl. Tracks Radiat. Meas. vol. 8, pp. 1-8, 1984.

[17] R. Katz, "Track Structure Interpretation of Heavy Ion Lithography in Relation to Radiobiology and the Structure of Etchable Tracks in Solid State Nuclear Track Detectors,", in Eighth Symposium on Microdosimetry, CEC, Julich, Germany, 1982.

[18] R. Katz, G. L. Sinclair, and M. P. R. Waligorski, "The Fricke Dosimeter as a 1-Hit Detector." Nucl. Tracks Radiat. Meas. vol. 11, pp. 301-307. 1986.

[19] R. Katz and G. R. Huang, "Track Core Effects in Heavy Ion Radiolysis," Radiat. Phys. Chem. vol.33, pp. 345-349, 1989.

[20] R. Katz, D. E. Dunn, and G. L. Sinclair, "Thindown in Radiobiology," Radiat. Prot. Dosim. vol. 13, pp. 281-284, 1985.

[21] R. Katz and S. C. Sharma, "Response of Cells to Fast Neutrons, Stopped Pions, and Heavy Ion Beams, Nucl. Instr. Meth. vol. 110, pp. 93-116, 1973.

[22] F. A. Cucinotta, R. Katz, J. W. Wilson, L. W. Townsend, J. E. Nealy, and J. L. Shinn, "Cellular Track Model of Biological Damage to Mammalias Cell Cultures from Galactic Cosmic Rays," NASA Technical Paper 3055, Feb. 1991.

[23] R. Katz and R. Zachariah, "E. Coli B. Modeled as a 1-Hit Detector," Ninth Int. Congress of Radiat. Res., Toronto, 1991.

[24] R. Katz and S. Wesely, "Cross Sections for Single and Double Strand Breaks in SV-40 Virus in EO Buffer after Heavy Ion Irradiation: Experiment and Theory," Radiat. and Environ. Biophys. vol. 30, pp. 81 85, 1991. 